\title{
Comparison of Radiographic and Clinical Results between MIPO Internal Fixation and Auxiliary Tension Suture Fixation for Proximal Humeral Fractures
}

X. YU', H. ZHANG 1 , R. J. XU1', A. Q. HUANG 1 , Z. H. YU' AND Y. MIAO'*

Department of Orthopedics, The Affiliated Suzhou Hospital of Nanjing Medical University, 26 Daoqian Street, Suzhou, 215000, P. R. China, ${ }^{1}$ Department of Orthopedics, Suzhou Municipal Hospital, 215000, Suzhou, P. R. China

Yu et al.: Comparison of MIPO alone or with Auxiliary Tension Suture Fixation

The aim of this study was to evaluate the efficacy of auxiliary tension suture fixation and minimally invasive percutaneous osteosynthesis internal fixation on proximal humeral fractures in patients. From May 2012 to May 2017, 66 patients with proximal humeral fractures were enrolled in the Affiliated Suzhou Hospital of Nanjing Medical University. A prospective follow-up cohort study was conducted on 47 patients with proximal humeral fractures, including 11 males and 36 females, average age $64.6 \mathrm{y}$, range 
27-92 y. According to the Neer's classification system, there were 10 two-part, 30 three-part and 7 four-part fractures. Twenty four patients were treated with minimally invasive percutaneous osteosynthesis internal fixation while 23 patients were managed with minimally invasive percutaneous osteosynthesis plus auxiliary tension suture fixation. Standard X-ray and physical examination post operation was performed. Consistant-visual analogue scale pain score, Constant-Murley score, University of California at Los Angeles score and disabilities of the arm, shoulder and hand score were comparable between the 2 groups. There were no significant differences between the 2 groups, however, a significantly greater supraspinatus force was observed in minimally invasive percutaneous osteosynthesis plus auxiliary tension suture fixation group compared to the minimally invasive percutaneous osteosynthesis alone group. Minimally invasive percutaneous osteosynthesis group had 2 cases of migration of great tuberosity and 1 case of acromial impingement. The auxiliary tension suture fixation group had 1 case of might valgus deformity and 1 case of fixation loosening and the complication rate was $12.5 \%$ of minimally invasive percutaneous osteosynthesis group compared to the 8.6 \% of auxiliary tension suture fixation group. Treatment of proximal humeral fractures with an auxiliary tension suture fixation was a good alternative to minimally invasive percutaneous osteosynthesis or conservative treatment, and should be performed routinely in displaced 2, 3 and 4-part fracturess.

Key words: Proximal humeral fractures, minimally invasive, auxiliary tension suture fixation

The proximal humerus fracture mainly refers to the proximal part of the surgical neck of the humerus, which usually involves the surgical neck, large and small nodules, anatomical neck or humeral head ${ }^{[1]}$. There is always controversy about its treatment. At present, the more commonly accepted indications for conservative treatment are, no significant displacement of the fracture end, stable fracture of the greater tibial tuberosity $<5 \mathrm{~mm}$ and more surgical treatment for the third and fourth part of the fracture. It accounts for $4-5 \%$ of all adult fractures ${ }^{[2-4]}$. The previous surgical treatment was mainly based on open reduction and internal fixation. However, with the increase of elderly patients, the understanding of proximal humerus fractures (PHF) and the improvement of functional requirements, the treatment concept has changed, the treatment effect is often not ideal, joint replacement technology is increasingly used in the treatment of senile, complex proximal humeral fractures ${ }^{[5]}$. In recent years, with the increase of traffic accidents, the incidence rate in young patients has also increased. At present, there is no effective guide for the treatment of PHF. Generally, according to the Neer classification, some fractures are treated conservatively. Two-part, 3-part and 4-part fractures require surgery ${ }^{[6]}$. It is often stated in the literature that 60 to $80 \%$ of nondisplaced or mildly displaced fractures can be treated conservatively. Nowadays, the new minimally invasive percutaneous osteosynthesis (MIPO) technique has been gradually applied ${ }^{[7,8]}$. The use of MIPO in the treatment of senile humeral shaft fractures meets the criteria for biological osteosynthesis with minimal incision to reduce iatrogenic soft tissue injury for fracture healing and early postoperative function. Exercise provides sufficient stability and the surgical approach is gradually becoming less invasive. However, simple plate fixation often fails to properly handle the traction of the rotator cuff on the large nodules, especially in patients with large nodular comminute fractures or fractures, which may lead to the displacement of the large nodules and cause limited shoulder movement. At the same time, due to the limitation of important vascular tissues such as the anterior tibialis anterior artery in the intramedullary interlamellar hiatus, the incision exposure range is limited ${ }^{[9-11]}$. If the traction is improper during operation, it may damage important blood vessels and nerves in the anterior tibial artery and axilla. If the fracture splits down or the posterior medial platform is involved, an incision improvement or other approach is required. Compared to intramedullary nailing in MIPO, MIPO rarely causes postoperative knee pain and is gradually accepted by more and more orthopaedic surgeons. Therefore, it is believed that the reconstruction of the rotator cuff function by non-absorbable suture technique has certain clinical significance ${ }^{[12-15]}$. The purpose of this study was to compare the clinical and radiographic outcomes of auxiliary tension suture fixation to MIPO in PHF. From May 2012 to May 2017, 
66 patients with PHFs were enrolled into the follow up study. All PHFs were classified according to the Neer classification, 47 patients aged 27-97 y were followed up (11 males and 36 females). Twenty four patients were managed with MIPO and the other 23 patients were managed with MIPO plus auxiliary tension suture fixation. Consistant-VAS pain score, Constant score, University of California at Los Angeles (UCLA) score, disabilities of the arm, shoulder and hand (DASH) score were used to evaluate the postoperative results, at the same time, the submuscular muscle strength was measured with a IDO dynamometer, proximal humeral lateral X-ray were performed 1, 2, 3, 6 and 12 mo after operation to observe bone union, including fracture angulation, displacement, internal fixation loose fracture, callus growth, intra-articular screw penetration at final follow-up, screw loosening, Axillary nerve injury, post-traumatic osteoarthritis, and avascular necrosis (AVN) were assessed during the follow-up period, then the medical records were checked to make some comparisons. All patients were given general anaesthesia and positioned in a beach chair position with an arm holder. MIPO group, after disinfection, towels were spread around the fracture, closed reduction was first used for 3-/4- part fractures. At the anterolateral of the acromion to distal $2 \mathrm{~cm}$, a longitudinal incision $3 \mathrm{~cm}$ was made, followed by layer by layer cutting. Blunt dissection using a periosteal elevator was carried out between the anterior deltoid and middle deltoid to arrive periosteum. Using K-wires to hold tuberculum, at the same time the shoulder joint was external rotated, reduction of fracture until a pleased result was obtained. A Philos plate with angle-stable screw fixation and radiolucent aiming device was used in all cases. After satisfied with the perspective position, proximal 4-5 locking screws, 2-3 distal locking screws were used for fixation, then radiolucent aiming device was used again to make sure that the location was right and stable.
In the MIPO plus auxiliary tension suture fixation group, the pre-steps were the same as the minimally invasive group, after K-wires were used to hold tuberculum; a non-absorbable suture was passed at supraspinatus, infraspinatus and tendinous insertion of rotator cuff respectively, then the suture was pulled together to form six pull wires. The plate was inserted into a channel which was separated from the operating area, the height of the plate was noted and a longitudinal incision of 2-3 cm was made after distal epidermal positioning to expose the anterior tibial bone. Suture was used to reduce large and small nodular fracture. After satisfied with the perspective position, dilute iodine and saline was used to clean the surgery area, on suture fascia and skin layers interruptedly. Dilute iodine and saline solution is a simple and inexpensive solution with the potential to prevent surgical site infection.

Independent student $t$-test was used to analyze continuous variables, and Chi-square test was used for dichotomous variables, to compare clinical outcomes between the two groups. Statistical analyses were performed with Statistical Product and Service Solutions (SPSS) 20.0 software (IBM, Armonk, NY, USA), $\mathrm{p}$ value of $<0.05$ was considered statistically significant.

The average follow-up time of all patients was 23.1 (12-33) mo. There were no statistically significant differences in terms of patient age, gender and Neer classification system between the two groups according to the Chi-square test (Table 1). According to the student- $T$ test (Table 2), there were no statistically significant differences in terms of fracture healing time, VAS score post-operation $1 \mathrm{w}$, VAS score post-operation $1 \mathrm{y}$, Constant-Murley score post-operation $1 \mathrm{y}$, UCLA score, DASH score, supraspinatus strength between the two groups. However, the operation time, ConstantMurley score, UCLA score, DASH score, supraspinatus

\section{TABLE 1: COMPARISON OF GENERAL DATA BETWEEN TWO GROUPS}

\begin{tabular}{|c|c|c|c|c|}
\hline Content & MIPO & Rotator cuff & Statistical data & $p$ value \\
\hline Gender (Male: Female) & $(5: 19)$ & $(6: 17)$ & $\chi^{2}=0.181$ & 0.671 \\
\hline Neer classification & & & $\chi^{2}=4.161$ & 0.125 \\
\hline II & 7 (29.2 \%) & $3(13.0 \%)$ & & \\
\hline III & $12(50 \%)$ & $18(78.3 \%)$ & & \\
\hline IV & $5(20.8 \%)$ & $2(8.7 \%)$ & & \\
\hline Injury mechanism & & & $\chi^{2}=0.119$ & 0.730 \\
\hline fall & 19 & 20 & & \\
\hline Car accident & 5 & 3 & & \\
\hline average age $(\mathrm{y})$ & $63.33(27-92)$ & $66.87(36-90)$ & $t=-0.947$ & 0.200 \\
\hline Average follow-up time (mo) & $23.28 \pm 9.22$ & $23.10 \pm 9.17$ & $t=0.568$ & 0.572 \\
\hline
\end{tabular}

$\chi^{2}$ (Chi-Square)

Special Issue 3, 2020 
strength 3 mo post-operation showed significant differences between the two groups $(p<0.05)$. In the MIPO group, two patient's showed loss of large tubercle of humerus reposition and 1 patient showed shoulder impact. There were no serious complications, like loose internal fixation, screws out, incision infection, radial nerve injury, with a rate of complications was $12.5 \%$. In the MIPO plus auxiliary tension suture fixation group, there was 1 patient with modest valgus deformity, loose internal fixation but there were no screws out, incision infection, radial nerve injury, humeral head necrosis, and the rate of complications was $8.6 \%$. T-test and Kruskal-Wallis test showed no significant difference between the immediate and 3-mo and 1-y postoperative neck angle imaging and clinical results
(Table 3). The shoulder joint is complicated compared to other joints among human upper limbs, the integrity of the rotator cuff muscles is crucial for stabilizing the rotation centre of the shoulder joint and assisting the deltoid muscle movement ${ }^{[16]}$. The rotator cuff provides $50 \%$ of the force required on the upper arm and $90 \%$ of the force required on the external rotation. Large and small nodules fracture displacement is more obvious in type III and IV proximal humeral fractures, however, there is the end of rotator cuff tendon and therefore, poor healing would result in dysfunction of rotator cuff tendons. According to the biomechanical test, the maximum contraction force of the human rotator cuff is about $340 \mathrm{~N}$, the humeral head can be pulled repeatedly when shoulder joint moves, it is easy to

TABLE 2: COMPARISON OF OBSERVATION DATA BETWEEN TWO GROUPS

\begin{tabular}{|c|c|c|c|}
\hline Content & MIPO & Rotator cuff & $P$ value \\
\hline Surgery time (min) & $81.17 \pm 27.55$ & $82.00 \pm 22.87$ & 0.911 \\
\hline \multicolumn{4}{|l|}{ VAS score } \\
\hline $1 \mathrm{w}$ & $5.33 \pm 1.09$ & $5.35 \pm 1.75$ & 0.973 \\
\hline $1 \mathrm{y}$ & $3.58 \pm 2.39$ & $2.87 \pm 1.91$ & 0.266 \\
\hline \multicolumn{4}{|l|}{ Constant-Murley score } \\
\hline $3 \mathrm{mo}$ & $49.42 \pm 21.73$ & $69.65 \pm 12.79$ & 0.055 \\
\hline $1 \mathrm{y}$ & $59.96 \pm 23.64$ & $70.61 \pm 12.57$ & 0.061 \\
\hline \multicolumn{4}{|l|}{ UCLA score } \\
\hline 3 mo & $21.92 \pm 7.77$ & $25.00 \pm 5.58$ & 0.127 \\
\hline $1 \mathrm{y}$ & $23.83 \pm 8.47$ & $26.48 \pm 4.86$ & 0.195 \\
\hline \multicolumn{4}{|l|}{ DASH score } \\
\hline $3 \mathrm{mo}$ & $50.25 \pm 20.44$ & $41.43 \pm 19.91$ & 0.141 \\
\hline $1 \mathrm{y}$ & $45.17 \pm 24.84$ & $39.39 \pm 17.29$ & 0.362 \\
\hline \multicolumn{4}{|c|}{ Supraspinatus muscle strength (kg) } \\
\hline $3 \mathrm{mo}$ & $1.17 \pm 0.645$ & $1.73 \pm 0.70$ & 0.007 \\
\hline $1 \mathrm{y}$ & $1.33 \pm 0.83$ & $1.87 \pm 0.67$ & 0.019 \\
\hline \multicolumn{4}{|l|}{ Forward angle $\left({ }^{\circ}\right)$} \\
\hline $3 \mathrm{mo}$ & $87.42 \pm 31.92$ & $97.43 \pm 26.78$ & 0.254 \\
\hline $1 \mathrm{y}$ & $94.21 \pm 31.57$ & $100.30 \pm 17.49$ & 0.416 \\
\hline \multicolumn{4}{|l|}{ Outreach $\left({ }^{\circ}\right)$} \\
\hline $3 \mathrm{mo}$ & $83.21 \pm 26.59$ & $98.65 \pm 27.33$ & 0.056 \\
\hline $1 \mathrm{y}$ & $90.58 \pm 31.21$ & $100.00 \pm 19.90$ & 0.223 \\
\hline \multicolumn{4}{|c|}{ Outward rotation activity score } \\
\hline $3 \mathrm{mo}$ & $4.38 \pm 1.996$ & $4.96 \pm 2.286$ & 0.357 \\
\hline $1 \mathrm{y}$ & $4.50 \pm 2.52$ & $5.70 \pm 2.12$ & 0.086 \\
\hline \multicolumn{4}{|c|}{ Internal rotation activity score } \\
\hline $3 \mathrm{mo}$ & $4.38 \pm 2.02$ & $5.30 \pm 1.30$ & 0.068 \\
\hline $1 \mathrm{y}$ & $4.50 \pm 1.84$ & $5.70 \pm 2.12$ & 0.292 \\
\hline Fracture healing time $(\mathrm{w})$ & $17.5(11-26)$ & $18.2(12-28)$ & 0.071 \\
\hline Complication (N) & 3 & 2 & 0.221 \\
\hline
\end{tabular}

TABLE 3: COMPARISON OF THE RADIOLOGICAL RESULTS BETWEEN TWO GROUPS

\begin{tabular}{lcccc}
\hline Immediately after surgery & $134.6^{\circ} \pm 7.8^{\circ}$ & $133.9^{\circ} \pm 6.5^{\circ}$ & $134.8^{\circ} \pm 8.0^{\circ}$ & $p=0.756$ \\
\hline three month after surgery & $133.2^{\circ} \pm 9.2^{\circ}$ & $131.4^{\circ} \pm 10.3^{\circ}$ & $134.0^{\circ} \pm 8.5^{\circ}$ & $p=0.223$ \\
One year after surgery & $131.5^{\circ} \pm 10.8^{\circ}$ & $125.8^{\circ} \pm 15.3^{\circ}$ & $132.9^{\circ} \pm 8.7^{\circ}$ & $p=0.156$ \\
\hline
\end{tabular}


cause humeral head inversion and even collapse ${ }^{[12,17]}$. There are multiple factors that can affect shoulder function after surgery, among the most common cause of postoperative shoulder joint dysfunction is poor postoperative position or poor healing of large nodules. According to biomechanical test, when the large nodule shift was $0.5 \mathrm{~cm}$, the required force for outreaching shoulder joints increased by $16 \%$, and when the shift was $1 \mathrm{~cm}$, it was increased by $27 \%{ }^{[18]}$. Plate and screw fixation are nowadays the most common way to treat the PHF. However, in order to avoid penetration into the joint cavity, single cortical fixation was always used, at the same time, this fixation is easy to pull out and the stability is not good ${ }^{[19]}$. Therefore, it is often necessary to brake after surgery to avoid the occurrence of related complications. However, several studies have shown that patients with postoperative rehabilitation who within 2 w suffered from poor final shoulder function ${ }^{[20]}$ and a significant displacement of the large nodules relative to the humeral shaft can occur even according to postoperative standard rehabilitation procedures for passive activities (figs. 1 and 2).

Therefore, in order to enhance the stability of proximal humeral fracture fixation and to facilitate early functional exercise ${ }^{[21]}$, the rotator cuff can be sutured to the locking plate reserved by non-absorbable sutures in addition to reconstructing the proximal medial tibial support ${ }^{[22]}$. Using non-absorbable suture for large nodules and surgical neck fractures of the humerus, clinical results showed excellent rate of recovery of $89 \%$, which also proved that the method has certain feasibility ${ }^{[23]}$. In terms of biomechanics, fresh cadaveric tests were used to determine the effect of three fixation methods on the stability of large tarsal tuberosity with the results confirmed that the biomechanical stability of the absorbable suture was better than that of screw

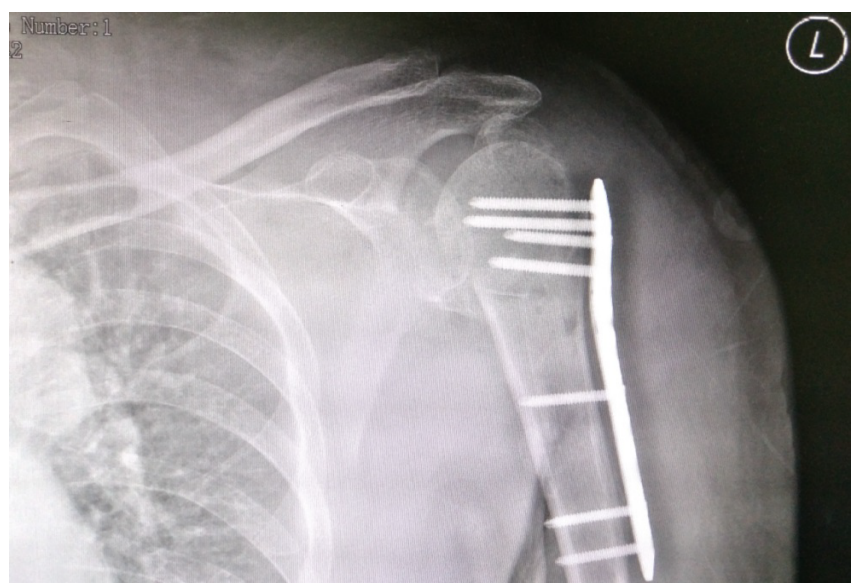

Fig. 1: Displaced CT image of proximal humerus fracture

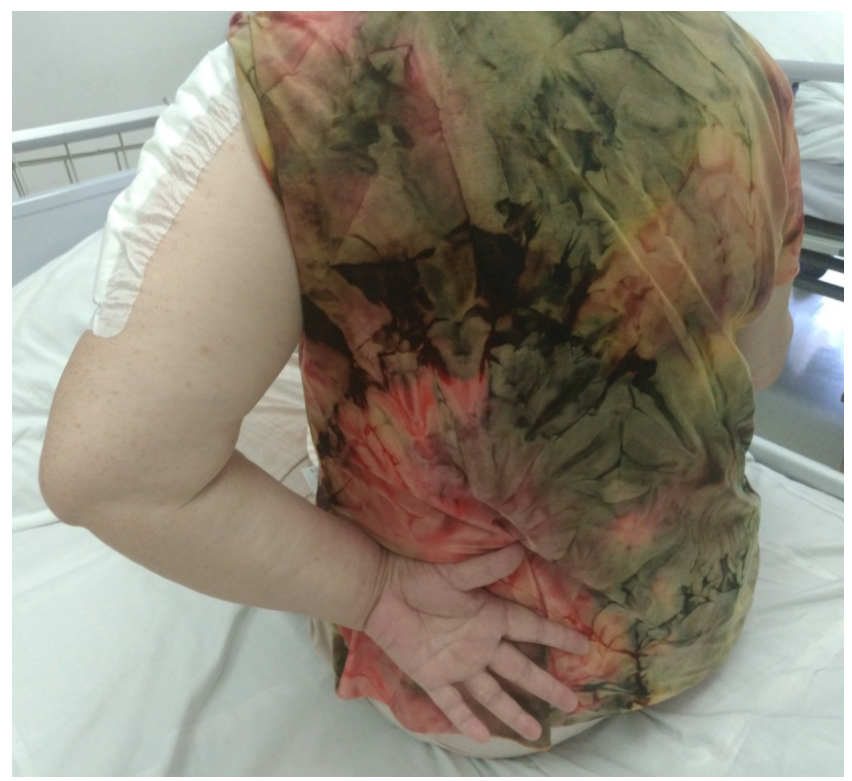

Fig. 2: Displacement of proximal humerus fracture

fixation, and it could effectively restore mechanical stability of large nodular fractures. The purpose of this study was to combine the MIPO technique with rotator cuff suture technique for PHFs and to evaluate the clinical efficacy and imaging results of minimally invasive locking plate and auxiliary rotator cuff suture in the treatment of proximal humerus fractures. In this study, high strength non-absorbable suture was used and the principle of metaphyseal ligament pull was fully utilised to reduce the damage to soft tissue and at the same time rebuild rotator cuff tension and improve its stability. It also avoided contracture of supraspinatus muscle, large and small round muscle after operation and promote the recovery of muscle strength, increase the degree of joint motion in the short term. According to the clinical and radiological results, the auxiliary rotator cuff suture group was better than the plate fixation group in all observation indicators in addition to VAS score $1 \mathrm{w}$ later, and there was a statistically significant difference in joint motion 3 mo after operation. The assisted rotator cuff suture group had a higher $1 \mathrm{w}$ after surgery VAS score, which was considered to be related to the local distraction after suture of the rotator cuff, such as weaving sutures and dispersing tension. Beside all conveniences, the limitation of this study is lack of adequate number of patient samples and large sample prospective study might help to obtain more valid data. Treatment of PHFs with an auxiliary tension suture fixation was preferential to minimally invasive percutaneous osteosynthesis or conservative treatment, and should be used in displaced 2, 3 and 4-part fractures. 


\section{Acknowledgements:}

The authors appreciate the support of The Affiliated Suzhou Hospital of Nanjing Medical University.

\section{Conflict of interest:}

All authors report no conflicts of interest in this work.

\section{REFERENCES}

1. Launonen AP, Lepola V, Saranko A, Flinkkila T, Laitinen M, Mattila VM. Epidemiology of proximal humerus fractures. Arch Osteoporos 2015;10: 209.

2. Clavert P, Hatzidakis A, Boileau P. Anatomical and biomechanical evaluation of an intramedullary nail for fractures of proximal humerus fractures based on tuberosity fixation. Clin Biomech 2016;32:108-12.

3. Mellano CR, Frank RM, Shin JJ, Jain A, Zuke WA, Mascarenhas $\mathrm{R}$, et al. Subpectoral Biceps Tenodesis With PEEK Interference Screw: A Biomechanical Analysis of Humeral Fracture Risk. Arthroscopy 2018;34:806-13.

4. Christiano A, Pean P, Konda S, Egol ZK. Functional outcome after proximal humerus fracture fixation: understanding the risk factors. Acta Orthop Belg 2017;82:1-7.

5. Chen AC, Chiu CH, Hsu KY, Chan YS. Influence of age, gender, and radiographic features on the deltoid splitting approach for surgical osteosynthesis in displaced proximal humerus fractures: a comparison study. BMC Musculoskelet Disord 2017;18:495.

6. Altintas B, Biber R, Bail HJ. Is it safe to assist proximal humeral nailing to residents? An analysis of 1134 cases. Injury 2016;47: S7-9.

7. Zhao W, Zhang Y, Johansson D, Chen X, Zheng F, Li L. Comparison of Minimally Invasive Percutaneous Plate Osteosynthesis and Open Reduction Internal Fixation on Proximal Humeral Fracture in Elder Patients: A Systematic Review and Meta-Analysis. Biomed Res Int 2017;2017:3431609.

8. Ortmaier R, Filzmaier V, Hitzl W, Bogner R, Neubauer T, Resch $\mathrm{H}$, et al. Comparison between minimally invasive, percutaneous osteosynthesis and locking plate osteosynthesis in 3-and 4-part proximal humerus fractures. BMC Musculoskelet Disord 2015;16:297.

9. Li L, Tian W. Displaced avulsion fractures of the posterior cruciate ligament: Treated by stellate steel plate fixation. Indian J Orthop 2015;49:171-5.

10. Yang CG, Ji WF, Ma ZC. Clinical effects of steel plate fixation for the failure of arthrodesis of ankle by screw fixation. Zhongguo Gu Shang 2012;25:639-41.

11. Yu LP, Dai GF, Duan JC, Xu YB, Chen WJ, Zhou LQ. Thin steel plate with thumbtack fixation in the treatment of massive presacral venous plexus hemorrhage. Chin Med J 2011;124:3180-1.
12. Wall KC, Toth AP, Garrigues GE. How to Use a Graft in Irreparable Rotator Cuff Tears: A Literature Review Update of Interposition and Superior Capsule Reconstruction Techniques. Curr Rev Musculoskelet Med 2018;11:122-30.

13. Sanchez G, Chahla J, Moatshe G, Ferrari MB, Kennedy NI, Provencher MT. Superior Capsular Reconstruction With Superimposition of Rotator Cuff Repair for Massive Rotator Cuff Tear. Arthrosc Tech 2017;6:e1775-9.

14. Sethi P, Franco WG. The Role of Superior Capsule Reconstruction in Rotator Cuff Tears. Orthop Clin North Am 2018;49:93-101.

15. Pfalzer F, Huth J, Sturmer E, Endele D, Kniesel B, Mauch F. Serial clinical and MRI examinations after arthroscopic rotator cuff reconstruction using double-row technique. Knee Surg Sports Traumatol Arthrosc 2017;25:2174-81.

16. Peltonen H, Arokoski J, Kallinen M, Pullinen T. Muscle loading and activation of the shoulder joint during humeral external rotation by pulley and variable resistance. J Electromyogr Kinesiol 2012;22:424-30.

17. Sproul RC, Iyengar JJ, Devcic Z, Feeley BT. A systematic review of locking plate fixation of proximal humerus fractures. Injury 2011;42:408-13.

18. Bono CM, Renard R, Levine RG, Levy AS. Effect of displacement of fractures of the greater tuberosity on the mechanics of the shoulder. J Bone Joint Surg Br 2001;83:105662.

19. Jia L, Wang TB, Zhou J, Huang W, Lu H, Jiang BG. Evaluation of the use of structure screw in PHILOS plate for treatment of proximal humerus fracture in Chinese. Beijing Da Xue Xue Bao Yi Xue Ban 2015;47:269-71.

20. Tanner MW, Cofield RH. Prosthetic arthroplasty for fractures and fracture-dislocations of the proximal humerus. Clin Orthop Relat Res 1983;179:116-28.

21. Gardner MJ, Weil Y, Barker JU, Kelly BT, Helfet DL, Lorich DG. The importance of medial support in locked plating of proximal humerus fractures. J Orthop Trauma 2007;21:185-91.

22. Park JY, An JW, Oh JH. Open intramedullary nailing with tension band and locking sutures for proximal humeral fracture: hot air balloon technique. J Shoulder Elbow Surg 2006;15:594-601.

23. Park MC, Murthi AM, Roth NS, Blaine TA, Levine WN, Bigliani LU. Two-part and three-part fractures of the proximal humerus treated with suture fixation. J Orthop Trauma 2003;17:319-25.

This is an open access article distributed under the terms of the Creative Commons Attribution-NonCommercial-ShareAlike 3.0 License, which allows others to remix, tweak, and build upon the work non-commercially, as long as the author is credited and the new creations are licensed under the identical terms

This article was originally published in a special issue, "Biomedical applications in Pharmaceutical Sciences" Indian J Pharm Sci 2020:82(2)Spl issue3;109-114 